\title{
In utero exposure to persistent organochlorine pollutants and reproductive health in the human male
}

\author{
Anne Vested ${ }^{1}$, Cecilia H Ramlau-Hansen ${ }^{1,2}$, Sjurdur F Olsen ${ }^{3}$, Jens Peter Bonde ${ }^{4}$, \\ Henrik Støvring ${ }^{5}$, Susanne L Kristensen ${ }^{1}$, Thorhallur I Halldorsson ${ }^{3,6}$, Panu Rantakokko ${ }^{7}$, \\ Hannu Kiviranta ${ }^{7}$, Emil $\mathrm{H}$ Ernst $^{8}$ and Gunnar Toft ${ }^{1}$
}

${ }^{1}$ Department of Occupational Medicine, Danish Ramazzini Centre, Aarhus University Hospital, Noerrebrogade 44 Building 2C, DK-8000 Aarhus C, Denmark, ${ }^{2}$ Section for Epidemiology, Department of Public Health, University of Aarhus, Bartholins Allé 2 Building 1260, DK-8000 Aarhus C, Denmark, ${ }^{3}$ Statens Serum Institut, Centre for Fetal Programming, Artellerivej 5, DK-2300 Copenhagen S, Denmark, ${ }^{4}$ Department of Occupational and Environmental Medicine, Bispebjerg Hospital of Copenhagen University, Bispebjerg Bakke 23 Building 33 1st floor, DK-2400 Copenhagen NV, Denmark, ${ }^{5}$ Department of Public Health, Biostatistics, Bartholins Allé 2 Building 1261, DK-8000 Aarhus C, Denmark, ${ }^{6}$ Faculty of Food Science and Nutrition, University of Iceland, Eiríksgata 29, 101 Reykjavík, Iceland, ' Department of Environmental Health, National Institute for Health and Welfare (THL), PO Box 95, FI-70701 Kuopio, Finland and ${ }^{8}$ Section West, Institute for Biomedicine, Aarhus University, Ole Worms Allé 2, build. 1170, 8000 Aarhus $C$, Denmark

Correspondence should be addressed to A Vested; Email: anneveed@rm.dk

\begin{abstract}
Persistent organochlorine pollutants (POPs) are ubiquitous, bioaccumulative compounds with potential endocrine-disrupting effects. They cross the placental barrier thereby resulting in in utero exposure of the developing fetus. The objective of this study was to investigate whether maternal serum concentrations of polychlorinated biphenyls (PCBs) and $p, p^{\prime}$-dichlorodiphenyldichloroethylene $\left(p, p^{\prime}\right.$-DDE) during pregnancy are associated with son's semen quality and reproductive hormone levels. During 2008-2009, we recruited 176 male offspring from a Danish cohort of pregnant women who participated in a study in 1988-1989. Each provided semen and blood samples that were analyzed for sperm concentration, total sperm count, motility, and morphology, and reproductive hormone levels, respectively. The maternal blood samples were collected in pregnancy week 30 and were analyzed for the concentrations of six PCBs (PCB-118, -138, -153, -156, -170, and -180) and $p, p^{\prime}$-DDE. The potential associations between in utero exposure to $\Sigma$ PCBs (pmol/ml), sdioxin like-(DL) PCBs (PCB-118 and -156) (pmol/ml), and $p, p^{\prime}$-DDE and semen quality and reproductive hormone levels were investigated using multiple regression. Maternal median (range) exposure levels of $\Sigma$ PCB, $\Sigma$ DL-PCB, and $p, p^{\prime}$-DDE were 10.0 (2.1-35.0) $\mathrm{pmol} / \mathrm{ml}, 0.8(0.2-2.7) \mathrm{pmol} / \mathrm{ml}$, and $8.0(0.7-55.3) \mathrm{pmol} / \mathrm{ml}$, respectively, reflecting typical background exposure levels in the late $1980 \mathrm{~s}$ in Denmark. Results suggested that in utero exposure to $\Sigma$ PCB, $\Sigma$ DL-PCB, and $p, p^{\prime}$-DDE was not statistically significantly associated with semen quality measures or reproductive hormone levels. Thus, results based on maternal PCB and $p, p^{\prime}$-DDE concentrations alone are not indicative of long-term consequences for male reproductive health; however, we cannot exclude that these POPs in concert with other endocrine-modulating compounds may have adverse effects.

Reproduction (2014) $\mathbf{1 4 8}$ 635-646
\end{abstract}

\section{Introduction}

Persistent organochlorine pollutants (POPs) such as polychlorinated biphenyls (PCBs) and organochlorine pesticides such as dichlorodiphenyltrichloroethane (DDT) are ubiquitous, organic pollutants that have been widely used until they were banned in Western countries in the late 1970s. Because of their lipophilic structure, they accumulate in adipose tissue and bioaccumulate in the food chain (Safe 1990, Cook et al. 2011) and, therefore, humans are at present mainly exposed to these POPs through diet, for example through consumption of fatty fish, dairy products, and meat (Gasull et al. 2011). The compounds are persistent and have long half-lives in human tissue (Wolff et al. 2000, Seegal et al. 2011), and can be detected in humans worldwide due to their wide distribution and usage. Many PCB congeners have been shown to display hormone-modulating activities, and the DDTdegradation product $p, p^{\prime}$-dichlorodiphenyldichloroethylene $\left(p, p^{\prime}\right.$-DDE) is regarded a potent androgen receptor antagonist (Kelce et al. 1995). Both groups of substances readily cross the placental barrier (Saxena et al. 1981, 
Jacobson et al. 1984), resulting in in utero exposure of the developing fetus. In addition, substantial postnatal exposure results from breastfeeding, whereby accumulated compounds are transferred from the mother to the infant.

Low sperm concentration is associated with a longer time to pregnancy and a sperm concentration of $40 \times 10^{6} / \mathrm{ml}$ is considered a threshold below which fecundity declines (Bonde et al. 1998). A recent study from an ongoing surveillance of semen quality in young Danish men reporting for military draft (1996-2010) has suggested that only around 23\% of young Danish men have an optimal sperm concentration of above $40 \times 10^{6} / \mathrm{ml}$ and more than $9 \%$ morphologically normal spermatozoa (Jørgensen et al. 2012). Furthermore, studies suggest that young Danish men, alongside young men from Norway and Germany, may have lower sperm concentrations than young men in other European countries (Jørgensen et al. 2002, Punab et al. 2002, Richthoff et al. 2002, Paasch et al. 2008, Axelsson et al. 2011, Fernandez et al. 2011). Together with indications of increasing incidences of testicular cancer and congenital malformations such as cryptorchidism and hypospadias, this has led to the hypothesis that exposure to endocrine-modulating compounds during fetal life could cause an imbalance in the fetal hormonal environment and affect normal development of male reproductive organs, thereby causing long-term effects on male reproductive health (Toppari et al. 1996, Toppari \& Skakkebaek 1998, Skakkebaek et al. 2001, Sharpe et al. 2003).

Cross-sectional studies have indicated an inverse association between serum levels of PCB153, PCB138, and $p, p^{\prime}$-DDE exposure in adult life and sperm motility (Rozati et al. 2002, Hauser et al. 2003, Richthoff et al. 2003, Toft et al. 2006), and studies of men living in areas sprayed with DDT have suggested inverse associations between $p, p^{\prime}$-DDE and sperm motility, semen volume, total sperm count, and positive associations with sperm tail defects and poor sperm chromatin condensation (De Jager et al. 2006, Aneck-Hahn et al. 2007).

In addition, the Yu Cheng oil-disease provided insights into the consequences of in utero exposure to high levels of PCBs. These included increased abnormal sperm morphology, decreased sperm motility, and reduced oocyte penetration capacity among in utero exposed cases (Guo et al. 2000).

Based on the hypotheses that fetal life exposures may affect male reproductive organ development and cause long-term effects on reproductive health, the aim of this study was to conduct a population-based follow-up study to investigate the potential effects of in utero exposure to background levels of PCBs and $p, p^{\prime}$-DDE on semen quality and reproductive hormone levels among young postpubertal Danish men born in the years 1988-1989. To our knowledge, this is the first population-based follow-up study to address the associations between in utero exposure to PCBs and $p, p^{\prime}$-DDE and semen quality and reproductive hormone levels in early adult life.

\section{Materials and methods \\ Population}

A Danish population-based pregnancy cohort was recruited in 1988-1989, when the pregnant women attended a routinely scheduled consultation with a midwife in Aarhus, Denmark, at pregnancy week 30. They answered a detailed questionnaire, were interviewed on dietary and lifestyle habits, and provided a blood sample for storage in a biobank. Of all 1212 pregnant women invited, 965 (80\%) participated (Olsen et al. 1995a, 1995b).

The follow-up for the male offspring of this cohort was initiated in 2008, where the sons were invited to fill in an internet-based questionnaire on health and lifestyle habits. In 2008-2009, the sons were invited to participate in a physical examination at Aarhus University Hospital, Denmark, where they provided a semen sample, a blood sample, and selfestimated testicular volume using an orchidometer. One hundred and seventy-six sons attended the physical examination, corresponding to a participation rate of $38 \%$ of all male offspring in the source cohort (Fig. 1). All participants signed a written informed consent before participation, and the study was approved by the regional ethics committee (registration number M-20010157).

Two participants were excluded from all statistical analyses due to azoospermia and abuse of anabolic steroids respectively. In addition, one participant was excluded due to an extremely high maternal serum level of PCBs and $p, p^{\prime}$-DDE (PCB-153 concentration: $12 \mathrm{ng} / \mathrm{ml}$ and $p, p^{\prime}$-DDE concentration: $32 \mathrm{ng} / \mathrm{ml}$ ) compared with the remaining participants, leaving a study population of 173 available for this study.

\section{Data collection}

The participants attended the physical examinations from February 2008 until September 2009. The young men were instructed to collect semen samples into plastic containers by

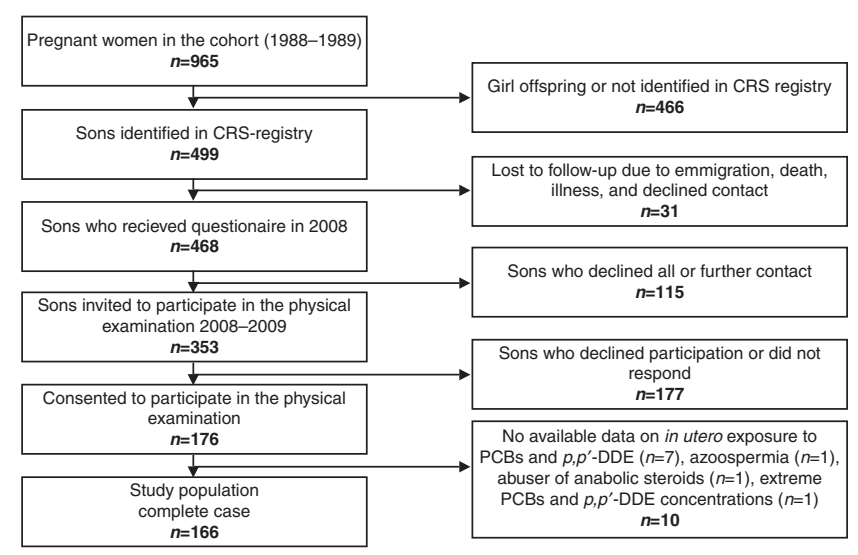

Figure 1 Flow chart for recruitment of participants. 
masturbation, at their residence or at the hospital, and to keep the samples close to the body during transport. At the hospital, semen samples were stored in a heating chamber $\left(37^{\circ} \mathrm{C}\right)$ until semen analysis. All participants reported time and date of the semen sample collection and information on potential spillage during semen sample collection. The participants were instructed to self-measure their testicular volumes by using a Prader orchidometer and finding the volume on the orchidometer, that best corresponded to the size of the testicle, going from the smallest towards the largest volume on the orchidometer. This method has previously been reported to be a valid method for measurement of testicular size (Ramlau-Hansen et al. 2007b). In addition, height and weight were measured for each participant by an examiner.

The blood samples were taken between 0730 and $1330 \mathrm{~h}$. The participants were instructed to present at the physical examination being in a fasted state for $10 \mathrm{~h}$.

\section{Semen analysis}

The semen analysis was initiated within $1 \mathrm{~h}$ from ejaculation for $86 \%$ of the participants and all semen samples were analyzed within $2 \mathrm{~h}$ from ejaculation. Conventional semen analysis was performed according to World Health Organization (WHO) guidelines from 1999 (World Health Organization 1999) and the Nordic Association for Andrology Manual on Basic Semen Analysis. The sperm concentration was assessed on two dilutions of the semen sample using a Neubauer counting chamber and the final sperm concentration calculated from the sum of the two counts divided by a factor derived from the dilution factor and the number of Neubauer counting chamber squares counted. Sperm motility was assessed in an aliquot of $6 \mu \mathrm{l}$ semen by classifying spermatozoa into four motility categories by counting the proportion of rapidly progressive ( $>25 \mu \mathrm{m} / \mathrm{s}$ ), slowly progressive (5-25 $\mu \mathrm{m} / \mathrm{s})$, non-progressive $(<5 \mu \mathrm{m} / \mathrm{s})$, and immotile spermatozoa for $2 \times 100$ spermatozoa. This was repeated in a new aliquot of $6 \mu \mathrm{l}$ semen and, hence, a total of 400 spermatozoa were classified per semen sample. Additional information is shown in the study reported by Vested et al. (2011). Furthermore, sperm concentration and motility were assessed by computerassisted semen analysis (CASA), using the Copenhagen Rigshospitalet Image House Sperm Motility Analysis System (CRISMAS) Clinical software version 4.6 (Image House Medical, IHMedical A/S, Copenhagen, Denmark), as previously described (Vested et al. 2011). For both conventional and CASA motility assessment, participants with missing information on motility assessments were assigned a mean of each of the motility categories based on the participants with information on sperm motility. The laboratory attended the Special Interest Group in Andrology ESHRE Subcommittee External Quality Assurance control program, and all tests were within the central $50 \%$ of the reference results. The sperm morphology was assessed using 'Strict criteria' (Menkveld et al. 1990). The assessments of morphology were only included in the statistical analyses for the participants where quality control status of the sperm morphology smears were in agreement with the 'strict criteria'.

\section{Blood sample analyses}

The maternal serum samples were collected in pregnancy week 30 in 1988-1989 and stored at $-20^{\circ} \mathrm{C}$ until analysis in 2011. The concentrations of $p, p^{\prime}$-DDE and six prevalent PCB congeners (PCB-118, -138,-153,-156, -170, and -180) were measured from $200 \mu \mathrm{l}$ aliquots of maternal serum by liquidliquid extraction followed by gas chromatography coupled with high resolution mass spectometry at the National Institute for Health and Welfare (THL), Department of Environmental Health, Kuopio, in Finland according to a sample pretreatment method described by Koponen et al. (2013). Two blanks and two control samples (NIST SRM 1589a) were included in each batch of samples $(n=25)$ in order to control for laboratory contamination and accuracy, and precision of the method respectively. The average recoveries of measured POPs in the control samples were $97-106 \%$ of the certified values. The between-assay coefficient of variation (CV) was $4.0 \%$ (at $0.17 \mathrm{ng} / \mathrm{ml}$ ) for PCB-118; $4.2 \%$ (at $0.54 \mathrm{ng} / \mathrm{ml}$ ) for PCB-138; $2.7 \%$ (at $0.97 \mathrm{ng} / \mathrm{ml}$ ) for PCB-153; $6.5 \%$ (at $0.08 \mathrm{ng} / \mathrm{ml}$ ) for PCB-156; 6.7\% (at $0.21 \mathrm{ng} / \mathrm{ml}$ ) for PCB-170; $2.6 \%$ (at $0.53 \mathrm{ng} / \mathrm{ml}$ ) for PCB-180; and $2.1 \%$ (at $11.2 \mathrm{ng} / \mathrm{ml}$ ) for $p, p^{\prime}$-DDE. The limits of quantification (LOQs) were between 2 and $5 \mathrm{pg} / \mathrm{ml}$. The concentrations of the six PCB congeners and $p, p^{\prime}-\mathrm{DDE}$ were above LOQ in all samples.

In offspring blood samples, serum concentrations of $\mathrm{LH}$, FSH, estradiol, and testosterone were analyzed using immunoassays (cobas 6000 e601, Roche Diagnostics) at the Department of Clinical Biochemistry, Aarhus University Hospital, Denmark with CVs being 1.1-2.4, 1.9-2.1, 1.5-2.9, and $2.2-4.5 \%$, respectively, and concentrations of sex hormone-binding globulin (SHBG) were measured using a solid-phase two-site chemiluminescent immunometric assay (IMMULITE 2000, Siemens Healthcare Diagnostics Products Ltd., Gwynedd, UK) with a CV of 4.5-4.7\%. Inhibin-B was measured undiluted using a commercially available ELISA (Oxford Bio-innovation Ltd, Oxfordshire, England, UK) with a detection of $20 \mathrm{pg} / \mathrm{ml}$ and a CV below $7 \%$ at the Laboratory of Reproductive Biology, Juliane Marie Centre for Women, Children and Reproduction, University Hospital of Copenhagen, Denmark. The measurements below the detection limit for estradiol $(n=3)$ were recoded to half the detection limit $(0.025 \mathrm{nmol} / \mathrm{l})$.

\section{Statistical analysis}

We converted each of the measured PCB congeners into molar concentrations by dividing PCBs in $\mathrm{pg} / \mathrm{ml}$ by the congener's molecular weight $(\mathrm{g} / \mathrm{mol})$, and PCB congeners were summed in two exposure groupings: i) $\Sigma$ all measured PCBs (pmol/ml) ( $\Sigma$ PCBs), and ii) $\Sigma$ dioxin-like PCBs (PCB-118 and PCB-156) ( $\Sigma \mathrm{DL}-\mathrm{PCBs})(\mathrm{pmol} / \mathrm{ml})$. In addition, $p, p^{\prime}$-DDE concentration in maternal blood samples was selected as a biomarker of organochlorine pesticide exposure and converted into molar concentration $(\mathrm{pmol} / \mathrm{ml})$. Each exposure variable was divided into three exposure groups (low, medium, and high) based on exposure tertiles. The outcome variables included semen parameters (sperm concentration, total sperm count, semen volume, percentage of progressive spermatozoa (rapidly 
progressive and slowly progressive), percentage of motile spermatozoa (rapidly progressive, slowly progressive, and non-progressive spermatozoa), and percentage of morphologically normal spermatozoa, mean right and left testicular volume, and reproductive hormones (testosterone, free testosterone calculated as suggested by Vermeulen et al. (1999), estradiol, LH, FSH, SHBG, and inhibin B).

The crude differences between tertile exposure groups and linear trends on continuous $\Sigma$ PCBs, $\Sigma$ DL-PCBs, and $p, p^{\prime}$-DDE concentrations and the studied outcomes (untransformed) were assessed by linear regression analysis with robust variance estimates. The adjusted trends were tested by multiple regression analysis, by entering the summed PCB levels or $p, p^{\prime}$-DDE levels as continuous variables.

The outcome variables were natural logarithm (In) transformed before multiple regression analysis, and low PCB groups or low $p, p^{\prime}$-DDE group were used as referents. The results from the multiple regression analyses are presented as relative differences on the original measurement scale with $95 \%$ confidence intervals $(95 \% \mathrm{Cl})$. All multiple regression results were adjusted for the history of reproductive tract disease (inguinal hernia, varicocele, testicular hydrocele, incarcerated hernia, phimosis, torsio testis, clamydia, gonorrhea, and epididymitis combined into a combined into one variable, yes/ no), sons' BMI, $\left(\mathrm{kg} / \mathrm{m}^{2}\right)$ (Sermondade et al. 2013), sons' smoking status (current and occasional smoker/ ex and never smoker) (Ramlau-Hansen et al. 2007c), maternal smoking during pregnancy (yes/ no) (RamlauHansen et al. 2007a), socioeconomic status (total annual income for the household in 1987 (<200000 DKK/ $\geq 200000$ DKK)), and maternal serum total lipid concentration (continuously in $\mathrm{g} / \mathrm{l}$ ). Total lipid concentration in maternal serum $(\mathrm{g} / \mathrm{l})$ was calculated using the following equation for estimation of total lipid based on the information on serum cholesterol and triglycerides: total lipid $=0.96+1.28 \times($ cholesterol $(g / l)+$ triglycerides $(g / l))$. For this calculation, the average molecular weights of cholesterol and triglycerides were assumed to be 571 and $807 \mathrm{~g} / \mathrm{mol}$, respectively, based on the assumption that the proportion of free and esterified cholesterol was 1:2 (Rylander et al. 2006). In addition, results on sperm concentration, total sperm count, percentage progressive spermatozoa, percentage motile spermatozoa, semen volume, and testicular volume were adjusted for abstinence time ( $\leq 48 \mathrm{~h} / \geq 49 \mathrm{~h})$; sperm concentration was adjusted for spillage during semen sample collection (yes/ no); percentage of progressive spermatozoa and percentage of motile spermatozoa were adjusted for time from ejaculation to semen analysis (continuous, minutes); and the reproductive hormones were adjusted for time of the day of blood sampling (0730-0929 h/0930-1129 h/ $\geq 1130 \mathrm{~h})$. The participants who reported spillage during semen sample collection were excluded from multiple regression analysis on total sperm count and semen volume. All statistical analyses were performed using Stata 12.1 software (Stata Corporation, College Station, TX, USA).

\section{Multiple imputation analyses}

Multiple imputation was used to handle missing data under the assumption that data fulfilled the missing at random criteria (Sterne et al. 2009).
The data on the exposure variables were missing on seven participants due to missing maternal blood samples or inadequate blood sample volume for POP concentration measurements. The numbers of participants with missing data in the outcome variables ranged between two and eight and the number of participants with missing values in the co-variates ranged between 0 and 15 .

For the main imputation model, 100 datasets were imputed with values for missing data. Before imputation, assumptions of normal distributions of the residuals of all continuous variables imputed on the linear scale in the multiple imputation model were checked and confirmed. We used the best transformations of the outcome variables for the multiple imputation of missing values (sperm concentration: cubic root; total sperm count, semen volume, and inhibin B: square root; progressive motility and all motility: squared; FSH: In; and morphologically normal spermatozoa, $\mathrm{LH}$, estradiol, testosterone, free testosterone, and SHBG; no transformation). Imputations for the main model were based on including all outcomes, exposure variables, and potential confounders mentioned above plus fever within the last three months (participants), maternal pre-pregnancy BMI, parity, maternal alcohol consumption-, maternal educational level, and maternal serum hexachlorbenzene level during pregnancy in the predictive models. In addition, interval censoring was allowed for variables that were imputed on a continuous scale according to plausible ranges predicted from the ranges within the dataset and limits of detection. All imputations and corresponding analyses were performed using Stata 12.1.

Sensitivity analyses with different imputation models were performed to check for consistency in results under different imputation models, but no discernible differences were observed in results (details available upon request).

\section{Results}

The 173 male offspring participating in the follow-up study had a median (range) age of 20 (19-21) years. Complete case data on maternal pregnancy week 30 serum concentrations $(\mathrm{ng} / \mathrm{ml})$ and lipid-adjusted serum concentrations (ng/g lipid) of $p, p^{\prime}-\mathrm{DDE}$, and the

Table 1 Maternal serum levels of six PCB congeners and $p, p^{\prime}$-DDE.

\begin{tabular}{lc}
\hline & Median (range) \\
\hline Wet weight serum concentrations (ng/ml) & \\
PCB-118 & $0.17(0.05-0.59)$ \\
PCB-138 & $0.75(0.14-2.58)$ \\
PCB-153 & $1.46(0.27-5.81)$ \\
PCB-156 & $0.10(0.02-0.34)$ \\
PCB-170 & $0.38(0.08-1.14)$ \\
PCB-180 & $0.71(0.15-2.44)$ \\
$p, p^{\prime}-D D E$ & $2.55(0.23-17.59)$ \\
Lipid-adjusted serum concentrations (ng/g lipid) & \\
PCB-118 & $20(6-60)$ \\
PCB-138 & $88(16-263)$ \\
PCB-153 & $165(31-591)$ \\
PCB-156 & $12(2-37)$ \\
PCB-179 & $43(9-148)$ \\
PCB-180 & $81(18-277)$ \\
$p, p^{\prime}-D D E$ & $288(31-2208)$ \\
\hline
\end{tabular}




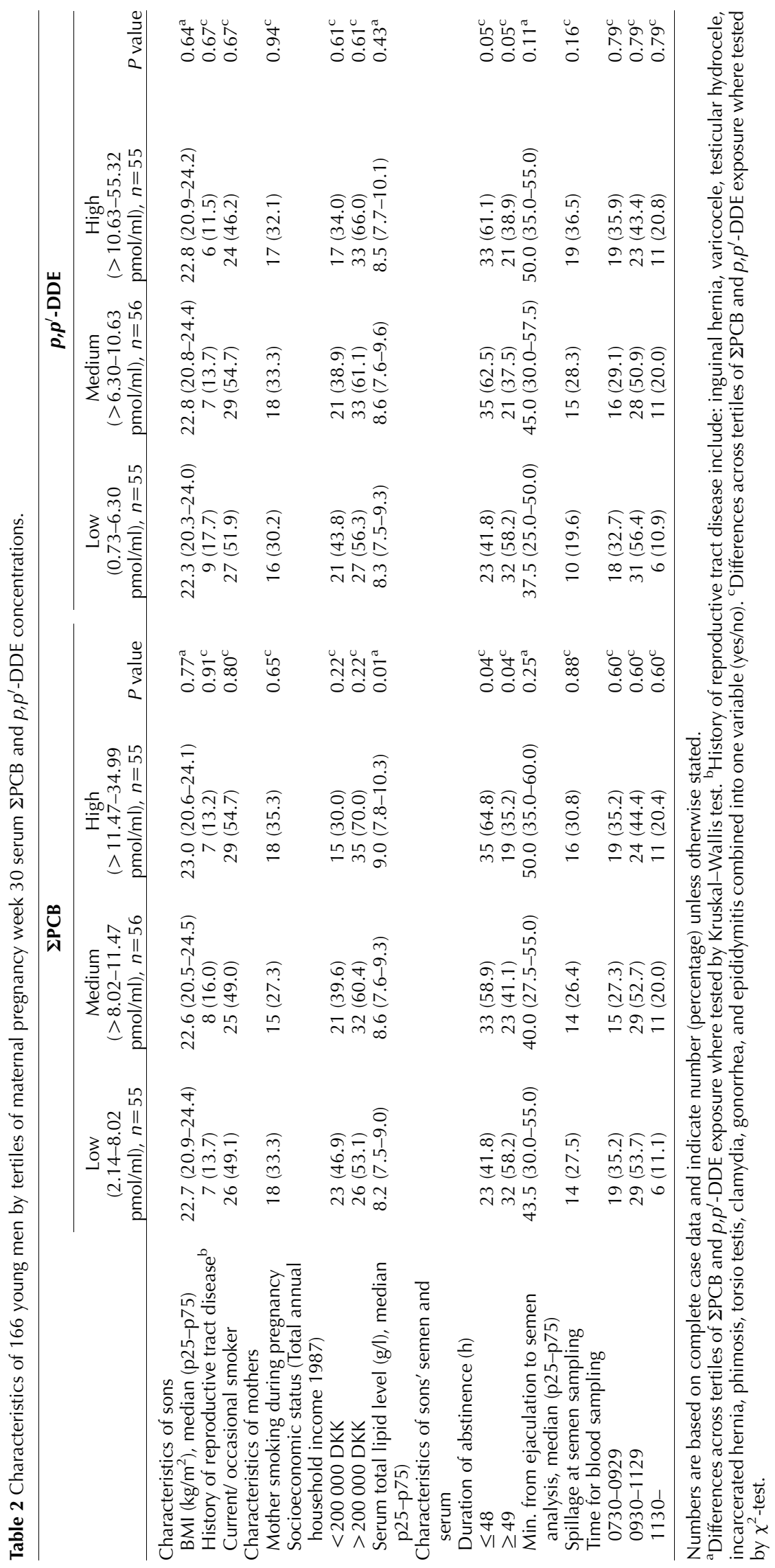




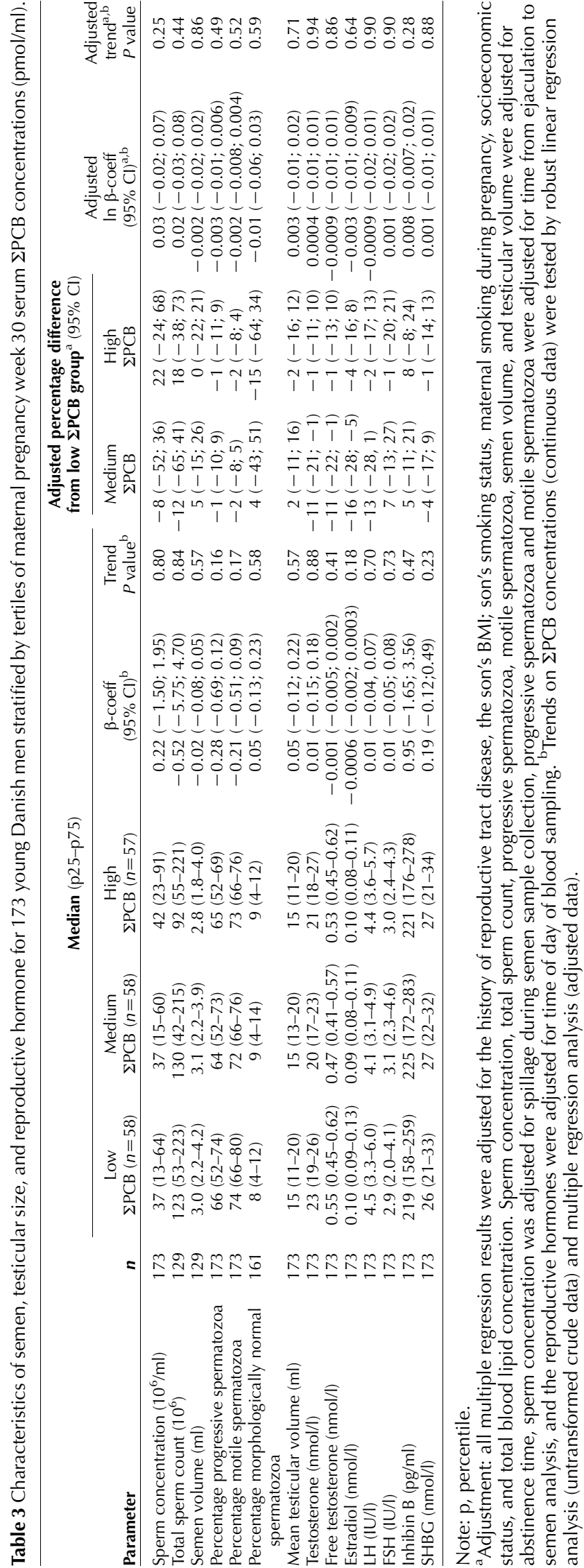

measured PCB congeners (PCB-118, -138, -153, -156, -170 , and -180 ) are given in Table 1, and characteristics of the participants according to tertiles of $\Sigma$ PCB and $p, p^{\prime}$-DDE exposure are shown in Table 2. Maternal serum lipid concentrations and sons' abstinence time differed substantially among the exposure groups. The mothers in the lowest $\Sigma$ PCB tertile had statistically significantly lower serum lipid levels than mothers in the highest tertile, and more participants in the lowest $\Sigma$ PCB- and $p, p^{\prime}$-DDE tertiles had been sexually abstinent for more than 2 days compared with the high $\Sigma$ PCB tertile and both medium and high $p, p^{\prime}$-DDE tertiles (Table 2).

The results from the main multiple imputation model showed no consistent associations between any of the exposure variables $\left(\Sigma \mathrm{PCB}, \Sigma \mathrm{DL}-\mathrm{PCB}\right.$, or $p, p^{\prime}$-DDE) and the studied outcomes for either crude or adjusted data (Tables 3, 4, and 5). However, in crude models, there were statistically significantly lower testosterone, free testosterone, estradiol, and $\mathrm{LH}$ levels in the medium $\Sigma$ PCB exposure group compared with the reference group (low), which were corroborated by the adjusted analyses, although not statistically significant for $\mathrm{LH}$ (Table 3). In addition, sons in the medium $p, p^{\prime}$-DDE exposure tertile had significantly lower semen volume and percentage of progressive spermatozoa compared with the reference group, but differences between the reference group and the sons exposed to the highest $p, p^{\prime}$-DDE tertile were not statistically significant. After adjustment, differences between the reference groups and the medium exposure groups attenuated and were no longer statistically significant. However, in the crude analyses, both the medium and the high tertile had significantly less percentage motile spermatozoa compared with the low $p, p^{\prime}$-DDE tertile. This was corroborated by the adjusted analyses with a significant difference between the high and the low tertiles and a tendency toward less spermatozoa in the medium tertile compared with the reference. None of the differences between groups were, however, corroborated by statistically significant trends on continuous exposures (Table 5).

There were no indications of threshold effects or doseresponse relationships between in utero exposure to $\Sigma \mathrm{PCB}, \Sigma \mathrm{DL}-\mathrm{PCB}$, and $p, p^{\prime}-\mathrm{DDE}$ and semen quality measures or reproductive hormone levels at the examined exposure ranges.

CASA results supported the results from the conventional semen analysis assessment (Supplementary Tables 1, 2 and 3, see section on supplementary data given at the end of this article). In addition, complete case analyses (Supplementary Tables 4, 5 and 6) and sensitivity analyses with different imputation models (fewer predictors, more predictors, and a model where the best transformation of outcomes and variables were used in stead of In-transformations in the multiple regression model) all supported the results from the main imputation model, except that the 'best transformation' model 


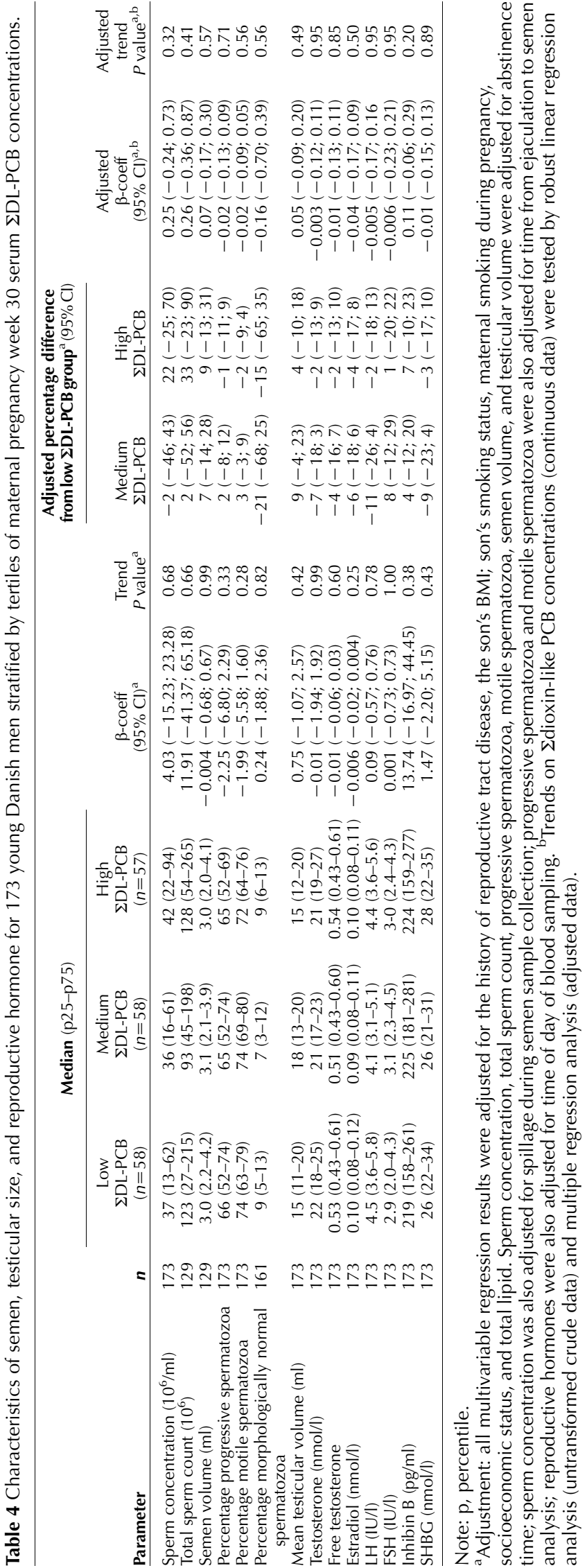

indicated a positive association between continuous $p, p^{\prime}$ DDE exposure and sperm concentration (Supplementary Table 7), which was even more pronounced in the complete case analyses (Supplementary Table 6). In addition, complete case analyses also suggested that higher in utero exposure to $p, p^{\prime}$-DDE was statistically, significantly associated with higher total sperm count in adult life (Supplementary Table 6).

When comparing PCB and $p, p^{\prime}$-DDE levels, according to level of participation in the study, there were no statistically significant differences in exposure levels between the sons who participated in the physical examination, those who only filled in questionnaires, and those who were lost to follow-up $(P=0.11, P=0.12$, and $P=0.48$ for $\Sigma$ PCB, $\Sigma$ DL-PCB, and $p, p^{\prime}-\mathrm{DDE}$, respectively; Table 6 ). Furthermore, there were no differences in maternal pre-pregnancy BMI, parity, social class, serum total lipid level, alcohol consumption during pregnancy or sons' BMI, or smoking status. There was a tendency toward a higher degree of young men who had a history of reproductive tract disease among those who participated in the physical examination compared with those who only filled in the questionnaire $(P=0.06)$. The mothers of sons who did not participate in follow-up were statistically significantly younger than mothers of sons who participated in the physical examination. In addition, we identified less reported smoking during pregnancy among the mothers whose sons participated in the physical examination compared with those who did not participate in follow-up (Table 6).

\section{Discussion}

In this study, we found that semen quality measures and reproductive hormone levels were not consistently, adversely related to in utero exposure to either $\Sigma$ PCBs, $\Sigma$ DL-PCBs, or $p, p^{\prime}$-DDE when using the approach of analysing one chemical/one chemical group at a time.

A study on mice exposed in utero and postnatally through lactation to a mixture of PCB-101 and PCB-118 to doses estimated to represent $0,1,10$, and $100 \mu \mathrm{g}$ $\mathrm{PCB} / \mathrm{kg}$ per day suggested that perinatal PCB exposure was associated with decreased testis weight and sperm viability but not with sperm concentration. At the highest PCB dose, a significant reduction in anogenital distance (AGD) was also observed compared with controls (Pocar et al. 2012). Another study, in which rat dams were treated with a single dose of $375 \mu \mathrm{g}$ PCB-118/kg on gestational day 6 found that in utero exposure to PCB118 was associated with increased AGD and reduced testis and epididymis weights in the male offspring. In addition, sperm concentration, daily sperm production, and sperm counts were significantly reduced compared with unexposed controls. In this study, exposure levels were $\sim 100$-fold higher than human exposure levels in breast milk (Kuriyama \& Chahoud 2004). 


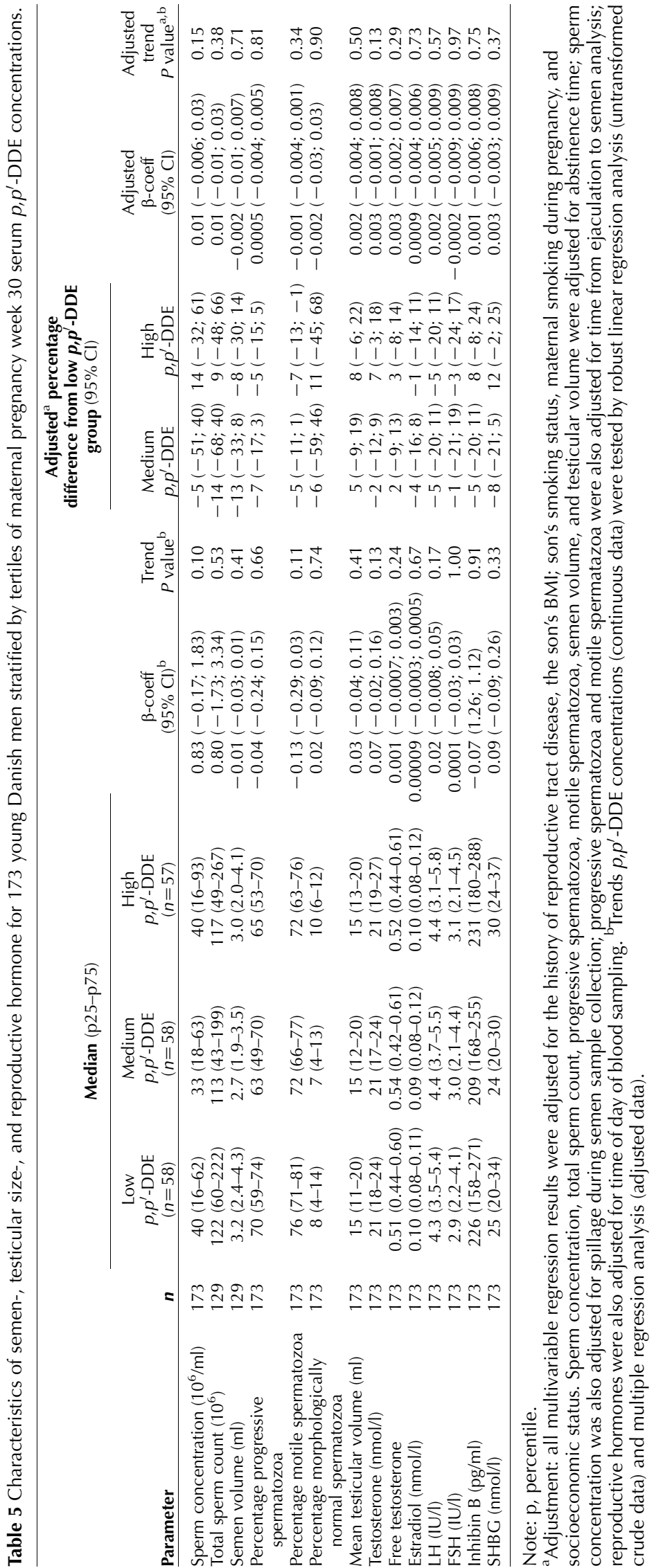


Table 6 Characteristics of mothers and sons at baseline (pregnancy week 30) and follow-up according to the level of participation.

\begin{tabular}{|c|c|c|c|c|}
\hline Characteristics & $\begin{array}{l}\text { Participating in physical } \\
\text { examination, } n=176\end{array}$ & $\begin{array}{c}\text { Filling in questionaire } \\
\text { only, } n=157\end{array}$ & $\begin{array}{c}\text { Not in follow-up, } \\
n=143\end{array}$ & $P$ value \\
\hline \multicolumn{5}{|l|}{ Maternal } \\
\hline$\Sigma$ PCBs $(\mathrm{pmol} / \mathrm{ml})$, median (p25-p75) & $10.1(7.3-12.9)$ & $10.4(7.6-13.0)$ & $9.0(6.6-12.2)$ & $0.12^{\mathrm{a}}$ \\
\hline इDioxin-like-PCBs $(\mathrm{pmol} / \mathrm{ml})$, median (p25-p75) & $0.8(0.6-1.1)$ & $0.8(0.6-1.1)$ & $0.8(0.6-1.0)$ & $0.11^{\mathrm{a}}$ \\
\hline$p, p^{\prime}$-DDE $(\mathrm{pmol} / \mathrm{ml})$, median $(\mathrm{p} 25-\mathrm{p} 75)$ & $8.2(5.2-12.4)$ & $7.3(4.9-11.2)$ & $8.0(5.1-11.9)$ & $0.48^{\mathrm{a}}$ \\
\hline Maternal age (years), mean (S.D.) & $29.6(4.4)$ & $29.0(4.2)$ & $28.3(4.5)$ & $0.03^{\mathrm{b}}$ \\
\hline Prepregnancy BMI $\left(\mathrm{kg} / \mathrm{m}^{2}\right)$, median (p25-p75) & $21.0(19.8-22.3)$ & $20.7(19.5-22.7)$ & $21.2(19.1-23.1)$ & $0.77^{\mathrm{a}}$ \\
\hline Nulliparous, $n(\%)$ & $112(64)$ & $96(61)$ & $83(58)$ & $0.60^{\mathrm{c}}$ \\
\hline Smoking during pregnancy, $n(\%)$ & $55(33)$ & $53(37)$ & $63(47)$ & $0.03^{\mathrm{c}}$ \\
\hline High social class, $n(\%)$ & $96(60)$ & $87(64)$ & $77(61)$ & $0.78^{\mathrm{c}}$ \\
\hline Blood total lipid level (g/l), median (p25-p75) & $8.6(7.7-9.6)$ & $8.8(7.9-10.0)$ & $8.7(7.9-9.6)$ & $0.33^{\mathrm{a}}$ \\
\hline Alcohol during pregnancy (g/day), median (p25-p75) & $2.0(0.4-4.6)$ & $1.5(0-4.4)$ & $1.5(0-3.5)$ & $0.53^{\mathrm{a}}$ \\
\hline \multicolumn{5}{|l|}{ Male offspring follow-up } \\
\hline BMI $\left(\mathrm{kg} / \mathrm{m}^{2}\right)$, median $(\mathrm{p} 25-\mathrm{p} 75)^{\mathrm{d}}$ & $22.2(20.5-24.1)$ & $22.4(20.7-24.4)$ & - & $0.50^{\mathrm{e}}$ \\
\hline Current/occasional smoker, $n(\%)$ & $83(50)$ & $66(43)$ & - & $0.24^{\mathrm{c}}$ \\
\hline History of reproductive tract disease, $n(\%)$ & $23(14)$ & $11(7)$ & - & $0.06^{\mathrm{c}}$ \\
\hline
\end{tabular}

${ }^{a}$ Kruskal-Wallis test. ${ }^{b}$ One-way ANOVA test. ${ }^{c} \chi^{2}$-test. ${ }^{d}$ BMI is calculated from anthropometric measures based on self-reported values. ${ }^{e}$ Wilcoxon rank-sum test.

We did not find any inverse associations between maternal pregnancy week 30 serum levels of PCB or $p, p^{\prime}$-DDE and testis size or sperm count. This supports previous epidemiological studies on associations between in utero exposure and male reproductive organ development, which generally found weak or no effects. Two nested case-control studies found that maternal pregnancy levels of $p, p^{\prime}$-DDE were not statistically significantly associated with increased risk of hypospadias and cryptorchidism (Longnecker et al. 2002, Bhatia et al. 2005), and two nested case-control studies on placental- and maternal serum concentrations of PCB during pregnancy found no increased risk of cryptorchidism (McGlynn et al. 2009, Virtanen et al. 2012). However, indications of increased risks of hypospadias and cryptorchidism in relation with in utero exposure to PCB and $p, p^{\prime}$-DDE have also been reported (Brucker-Davis et al. 2008, McGlynn et al. 2009, Rignell-Hydbom et al. 2012). We cannot exclude that the lack of statistically significant associations in the current study might be due to a lack of exposure contrast in the data. However, median maternal serum POP levels in our study were comparable with those of Rignell-Hydbom et al. (2012), who found a tendency toward an association with $p, p^{\prime}$-DDE levels. The data collection period in the Rignell-Hydbom study spans from 1986 until 2002, while maternal POP levels during pregnancy in the studies by Longnecker et al. (2002), Bhatia et al. (2005), and McGlynn et al. (2009) were substantially higher than the present concentrations, corresponding to a heavier POP load during the 1960s when specimens for these studies were collected.

The study by Guo et al. (2000) on young males prenatally exposed to high levels of PCBs and polychlorinated dibenzofurans (PCDFs) as a result of the Yu Cheng oil-disease suggested long-term adverse effects on sperm quality in terms of abnormal morphology, decreased sperm motility, and reduced oocyte penetration capacity (Guo et al. 2000).

We did not find any consistent associations between in utero exposure to $\mathrm{PCBs}$ or $p, p^{\prime}$-DDE and sperm morphology or sperm motility in the present study, except from the statistically significant less percentage motile spermatozoa in the high $p, p^{\prime}$-DDE tertile compared with the reference tertile, which was not corroborated by a significant trend on the continuous exposure. The males born to exposed Yu Cheng women were exposed to high levels of PCBs and PCDFs during fetal development because of their mothers' accidental ingestion of cooking oil contaminated with these POPs. As the young men in our cohort were only exposed to background POP levels in the late 1980s, differences between the findings of the Guo et al. (2000) study and our study are likely to be explained by differences in exposures levels.

Another study investigated the possible male reproductive health consequences of perinatal exposure to dioxins in a cohort of men born to mothers who were exposed to dioxins as a result of the trichlorophenol plant explosion near Seveso, Italy in 1976. This study found that sons who were exposed both in utero and through breast feeding had significantly lower sperm concentration, total sperm count, progressive motility, and total motile count than the unexposed controls. There were no differences in semen quality measures between sons, who were only exposed in utero, and controls (Mocarelli et al. 2011). In our study, we observed no associations between the dioxin-like PCBs (PCB-118 and PCB-156) and any of the investigated male reproductive health outcomes. This could be due to differences in exposure levels between the two studies or to a higher contribution by postnatal exposure, which was not assessed in the present study.

During the last decade, more focus has been turned toward "the mixture effect" - that compounds with 
potential hormone-modulating effects may only contribute with minor effects at the individual compound level, and that a health effect may only appear in concert with other chemicals. Animal studies have suggested that exposure to a mixture of different compounds can produce effects on male reproductive health at concentrations where there are no associations between the individual compounds and the male reproductive health outcome (Christiansen et al. 2008, Kortenkamp 2008), and studies have indicated that the mixture of compounds rather than individual chemical exposures may contribute to effects on male reproductive health (Krysiak-Baltyn et al. 2012). With the mixture effect in mind, we cannot exclude that PCB and $p, p^{\prime}-\mathrm{DDE}$ exposure in concert with other potential hormonemodulating compounds may result in adverse associations with male reproductive health, although overall no statistically consistent significant results were found in the current study using the single chemical/chemical group approach.

Complete case results and the multiple regression analyses, in which we checked In-transformed results with a model using the most optimal transformations of the outcome variables, suggested a positive association between maternal serum levels of $p, p^{\prime}$-DDE during pregnancy and the sperm concentration of sons in adult life. Similar findings have been reported independently in subsets of the study populations of two studies assessing the effects of adult male serum levels of $p, p^{\prime}$ DDE on male reproductive health (Toft et al. 2006, Haugen et al. 2011). Due to the antiandrogenic properties of $p, p^{\prime}$-DDE, this was opposite of what we expected and may be a result of multiple testing.

A previous study on this cohort of young men found inverse associations between maternal pregnancy week 30 serum levels of perfluorooctanoic acid (PFOA) and sperm concentration and total sperm count and positive associations to LH and FSH (Vested et al. 2013). A subanalysis adjusting for continuous PFOA did not alter the directions or significance levels of the results for either $\Sigma \mathrm{PCB}, \Sigma \mathrm{DL}-\mathrm{PCB}$, or $p, p^{\prime}$-DDE analyses (data not shown).

This study is to our knowledge the first population-based follow-up study to report on associations between in utero exposure to PCBs and $p, p^{\prime}$-DDE and semen quality and reproductive hormone levels in early adult life.

The longitudinal design of the present study has a major strength compared to cross-sectional studies attempting to study the same hypothesis. It allowed us to estimate the effects of exposure to POPs during a proposed critical window of exposure to exogenous compounds with semen quality measures and reproductive hormone levels later in life.

A possible limitation of this study includes the risk of selection bias, which could be present if study participation was associated with both POP exposure and reproductive outcomes. It has been shown by former studies that men experiencing fertility problems are more willing to participate in studies investigating male reproductive health outcomes (Bonde et al. 1996). The participants in this cohort were young and for the most part had no reproductive experience. Thus, it is unlikely that participation in this study was related to fecundity. However, there was a tendency toward more participants having a history of reproductive tract disease among the participants who attended the physical examination compared with the young men who only filled in the internet based questionnaire $(P=0.06)$. However, there is no reason to believe that a potential oversampling of young men with a history of reproductive tract disease would be related to exposure and thus unlikely that this would have biased the results. Participation rate of the physical examination was $38 \%$. However, losses to follow-up were not associated with $\Sigma \mathrm{PCB}, \Sigma \mathrm{DL}-\mathrm{PCB}$, or $p, p^{\prime}$-DDE exposure levels as there were no differences in exposure levels between the sons who participated in the physical examination, those who only filled in internet-based questionnaires, and those who did not participate in the follow-up study (Table 6). In addition, sons were unaware of their mothers' serum POP levels during pregnancy. Hence, we do not believe that selection bias is a major concern.

Because of the long half-lives of POPs in the human body, one blood sample measurement in pregnancy week 30 is considered a satisfactory proxy for fetal exposure during pregnancy.

A median lipid-adjusted $p, p^{\prime}$-DDE level of $288 \mathrm{ng} / \mathrm{g}$ lipid as measured in serum samples from pregnancy week 30 in 1988-1989 in the current study is comparable with $p, p^{\prime}$-DDE levels measured in male partners of infertility couples in USA in 2000-2001 (275 ng/g lipid) (Hauser et al. 2003), and considerably higher compared with Scandinavian levels measured in Northern and Southern Norway in 2001 (66-81 ng/g lipid; Haugen et al. 2011). The median PCB-153 lipidadjusted concentration was $165 \mathrm{ng} / \mathrm{g}$ lipid in the current study, which is somewhat higher than American and Scandinavian background levels in the 2000s (44-68 ng/g lipid; Hauser et al. 2003, Richthoff et al. 2003, Haugen et al. 2011). Thus, our findings are potentially relevant to contemporary generations.

Despite indications from previous studies of associations between POP levels in adult life and male reproductive health, the present results do not suggest long-term effects of in utero exposure to PCBs or $p, p^{\prime}$ DDE alone on semen quality measures or reproductive hormone levels; however it cannot be excluded that exposure to $\mathrm{PCBs}$ and $p, p^{\prime}$-DDE in combination with other endocrine-modulating compounds may have adverse effects on the male reproductive system.

\section{Supplementary data}

This is linked to the online version of the paper at http://dx.doi. org/10.1530/REP-13-0488. 


\section{Declaration of interest}

The authors declare that there is no conflict of interest that could be perceived as prejudicing the impartiality of the research reported.

\section{Funding}

The study was funded by the Danish council for Independent Research (271-05-0296) (09-073028) (09-065631), the Danish Council for Strategic Research (09-067124 (Centre for Fetal Programming), 09-063072, 2101-06-0005), the Danish Ministry of the Interior and Health, Research Centre for Environmental Health's Fund (7-302-02-6/3), the Aarhus University Research Foundation, the Frimodt-Heineke Foundation, and the Health Research Fund of Central Denmark Region.

\section{Acknowledgements}

The authors thank B. Brixen Joergensen, K Fredborg, and S Eeg Schoeler for recruiting and examining study participants and for the skillful assessments of semen samples. They also thank the technical staff at THL, Department of Environmental Health for POP analyses of the serum samples.

\section{References}

Aneck-Hahn NH, Schulenburg GW, Bornman MS, Farias P \& de Jager C 2007 Impaired semen quality associated with environmental DDT exposure in young men living in a malaria area in the Limpopo Province, South Africa. Journal of Andrology 28 423-434. (doi:10.2164/jandrol. 106.001701)

Axelsson J, Rylander L, Rignell-Hydbom A \& Giwercman A 2011 No secular trend over the last decade in sperm counts among Swedish men from the general population. Human Reproduction 26 1012-1016. (doi:10.1093/humrep/der045)

Bhatia R, Shiau R, Petreas M, Weintraub JM, Farhang L \& Eskenazi B 2005 Organochlorine pesticides and male genital anomalies in the child health and development studies. Environmental Health Perspectives 113 220-224. (doi:10.1289/ehp.7382)

Bonde JP, Giwercman A \& Ernst E 1996 Identifying environmental risk to male reproductive function by occupational sperm studies: logistics and design options. Occupational and Environmental Medicine 53 511-519. (doi:10.1136/oem.53.8.511)

Bonde JP, Ernst E, Jensen TK, Hjollund NH, Kolstad H, Henriksen TB, Scheike T, Giwercman A, Olsen J \& Skakkebaek NE 1998 Relation between semen quality and fertility: a population-based study of 430 first-pregnancy planners. Lancet 352 1172-1177. (doi:10.1016/S01406736(97)10514-1)

Brucker-Davis F, Wagner-Mahler K, Delattre I, Ducot B, Ferrari P, Bongain A, Kurzenne JY, Mas JC, Fenichel P \& Cryptorchidism Study Group from Nice Area 2008 Cryptorchidism at birth in Nice area (France) is associated with higher prenatal exposure to PCBs and DDE, as assessed by colostrum concentrations. Human Reproduction $\mathbf{2 3}$ 1708-1718. (doi:10.1093/humrep/den186)

Christiansen S, Scholze M, Axelstad M, Boberg J, Kortenkamp A \& Hass U 2008 Combined exposure to anti-androgens causes markedly increased frequencies of hypospadias in the rat. International Journal of Andrology 31 241-248. (doi:10.1111/j.1365-2605.2008.00866.x)

Cook MB, Trabert B \& McGlynn KA 2011 Organochlorine compounds and testicular dysgenesis syndrome: human data. International Journal of Andrology 34 e68-e84 discussion e84-5. (doi:10.1111/j.1365-2605. 2011.01171.x)

De Jager C, Farias P, Barraza-Villarreal A, Avila MH, Ayotte P, Dewailly E, Dombrowski C, Rousseau F, Sanchez VD \& Bailey JL 2006
Reduced seminal parameters associated with environmental DDT exposure and $p, p^{\prime}$-DDE concentrations in men in Chiapas, Mexico: a cross-sectional study. Journal of Andrology 27 16-27. (doi:10.2164/ jandrol.05121)

Fernandez MF, Duran I, Olea N, Avivar C, Vierula M, Toppari J, Skakkebaek NE \& Jørgensen N 2011 Semen quality and reproductive hormone levels in men from Southern Spain. International Journal of Andrology 35 1-10. (doi:10.1111/j.1365-2605.2010.01131.x)

Gasull M, de Basea MB, Puigdomènech E, Pumarega J \& Porta M 2011 Empirical analyses of the influence of diet on human concentrations of persistent organic pollutants: a systematic review of all studies conducted in Spain. Environment International 37 1226-1235. (doi:10. 1016/j.envint.2011.05.008)

Guo YL, Hsu PC, Hsu CC \& Lambert GH 2000 Semen quality after prenatal exposure to polychlorinated biphenyls and dibenzofurans. Lancet 356 1240-1241. (doi:10.1016/S0140-6736(00)02792-6)

Haugen TB, Tefre T, Malm G, Jonsson BA, Rylander L, Hagmar L, Bjorsvik C, Henrichsen T, Saether T, Figenschau Y et al. 2011 Differences in serum levels of CB-153 and $p, p^{\prime}-\mathrm{DDE}$, and reproductive parameters between men living south and north in Norway. Reproductive Toxicology 32 261-267. (doi:10.1016/j.reprotox.2011.06.072)

Hauser R, Chen Z, Pothier L, Ryan L \& Altshul L 2003 The relationship between human semen parameters and environmental exposure to polychlorinated biphenyls and $p, p^{\prime}$-DDE. Environmental Health Perspectives 111 1505-1511. (doi:10.1289/ehp.6175)

Jacobson JL, Fein GG, Jacobson SW, Schwartz PM \& Dowler JK 1984 The transfer of polychlorinated biphenyls (PCBs) and polybrominated biphenyls (PBBs) across the human placenta and into maternal milk. American Journal of Public Health 74 378-379. (doi:10.2105/AJPH.74. 4.378)

Jørgensen N, Carlsen E, Nermoen I, Punab M, Suominen J, Andersen AG, Andersson AM, Haugen TB, Horte A, Jensen TK et al. 2002 East-West gradient in semen quality in the Nordic-Baltic area: a study of men from the general population in Denmark, Norway, Estonia and Finland. Human Reproduction 17 2199-2208. (doi:10.1093/humrep/17.8.2199)

Jørgensen $N$, Joensen UN, Jensen TK, Jensen MB, Almstrup K, Olesen IA, Juul A, Andersson AM, Carlsen E, Petersen JH et al. 2012 Human semen quality in the new millennium: a prospective cross-sectional populationbased study of 4867 men. BMJ Open 2 1-13. (doi:10.1136/bmjopen2012-000990)

Kelce WR, Stone CR, Laws SC, Gray LE, Kemppainen JA \& Wilson EM 1995 Persistent DDT metabolite $p, p^{\prime}$-DDE is a potent androgen receptor antagonist. Nature 375 581-585. (doi:10.1038/375581a0)

Koponen J, Rantakokko P, Airaksinen R \& Kiviranta H 2013 Determination of selected perfluorinated alkyl acids and persistent organic pollutants from a small volume human serum sample relevant for epidemiological studies. Journal of Chromatography. A 1309 48-55. (doi:10.1016/j. chroma.2013.07.064)

Kortenkamp A 2008 Low dose mixture effects of endocrine disrupters: implications for risk assessment and epidemiology. International Journal of Andrology 31 233-240. (doi:10.1111/j.1365-2605.2007. 00862.x)

Krysiak-Baltyn K, Toppari J, Skakkebaek NE, Jensen TS, Virtanen HE, Schramm KW, Shen H, Vartiainen T, Kiviranta H, Taboureau $O$ et al. 2012 Association between chemical pattern in breast milk and congenital cryptorchidism: modelling of complex human exposures. International Journal of Andrology 35 294-302. (doi:10.1111/j.13652605.2012.01268.x)

Kuriyama SN \& Chahoud I 2004 In utero exposure to low-dose 2,3',4,4', 5-pentachlorobiphenyl (PCB 118) impairs male fertility and alters neurobehavior in rat offspring. Toxicology 202 185-197. (doi:10.1016/ j.tox.2004.05.006)

Longnecker MP, Klebanoff MA, Brock JW, Zhou H, Gray KA, Needham LL \& Wilcox AJ 2002 Maternal serum level of 1,1-dichloro-2,2-bis ( $p$-chlorophenyl)ethylene and risk of cryptorchidism, hypospadias, and polythelia among male offspring. American Journal of Epidemiology 155 313-322. (doi:10.1093/aje/155.4.313)

McGlynn KA, Guo X, Graubard BI, Brock JW, Klebanoff MA \& Longnecker MP 2009 Maternal pregnancy levels of polychlorinated biphenyls and risk of hypospadias and cryptorchidism in male offspring. Environmental Health Perspectives 117 1472-1476. (doi:10.1289/ehp. 0800389) 
Menkveld R, Stander FS, Kotze TJ, Kruger TF \& van Zyl JA 1990 The evaluation of morphological characteristics of human spermatozoa according to stricter criteria. Human Reproduction 5 586-592.

Mocarelli P, Gerthoux PM, Needham LL, Patterson DG Jr, Limonta G, Falbo R, Signorini S, Bertona M, Crespi C, Sarto C et al. 2011 Perinatal exposure to low doses of dioxin can permanently impair human semen quality. Environmental Health Perspectives 119 713-718. (doi:10.1289/ ehp.1002134)

Olsen SF, Hansen HS, Sandstrom B \& Jensen B 1995a Erythrocyte levels compared with reported dietary intake of marine n-3 fatty acids in pregnant women. British Journal of Nutrition 73 387-395. (doi:10.1079/ BJN19950041)

Olsen SF, Hansen HS, Secher NJ, Jensen B \& Sandstrom B 1995b Gestation length and birth weight in relation to intake of marine $\mathrm{n}-3$ fatty acids. British Journal of Nutrition 73 397-404. (doi:10.1079/BJN19950042)

Paasch U, Salzbrunn A, Glander HJ, Plambeck K, Salzbrunn H, Grunewald S, Stucke J, Vierula M, Skakkebaek NE \& Jørgensen N 2008 Semen quality in sub-fertile range for a significant proportion of young men from the general German population: a co-ordinated, controlled study of 791 men from Hamburg and Leipzig. International Journal of Andrology 31 93-102. (doi:10.1111/j.1365-2605.2007.00860.x)

Pocar P, Fiandanese N, Secchi C, Berrini A, Fischer B, Schmidt JS, Schaedlich K, Rhind SM, Zhang Z \& Borromeo V 2012 Effects of polychlorinated biphenyls in CD-1 mice: reproductive toxicity and intergenerational transmission. Toxicological Sciences 126 213-226. (doi:10.1093/toxsci/kfr327)

Punab M, Zilaitiene B, Jørgensen N, Horte A, Matulevicius V, Peetsalu A \& Skakkebaek NE 2002 Regional differences in semen qualities in the Baltic region. International Journal of Andrology 25 243-252. (doi:10. 1046/j.1365-2605.2002.00359.x)

Ramlau-Hansen CH, Thulstrup AM, Storgaard L, Toft G, Olsen J \& Bonde JP 2007 a Is prenatal exposure to tobacco smoking a cause of poor semen quality? A follow-up study American Journal of Epidemiology $\mathbf{1 6 5}$ 1372-1379. (doi:10.1093/aje/kwm032)

Ramlau-Hansen CH, Thulstrup AM, Bonde JP \& Ernst E 2007b Is selfmeasuring of testicular volume by a Prader orchidometer a valid method? Fertility and Sterility 87 1480-1482. (doi:10.1016/j.fertnstert.2006.11.032)

Ramlau-Hansen $\mathrm{CH}$, Thulstrup AM, Aggerholm AS, Jensen MS, Toft G \& Bonde JP 2007 C Is smoking a risk factor for decreased semen quality? A cross-sectional analysis Human Reproduction 22 188-196. (doi:10. 1093/humrep/del364)

Richthoff J, Rylander L, Hagmar L, Malm J \& Giwercman A 2002 Higher sperm counts in Southern Sweden compared with Denmark. Human Reproduction 17 2468-2473. (doi:10.1093/humrep/17.9.2468)

Richthoff J, Rylander L, Jonsson BA, Akesson H, Hagmar L, Nilsson-Ehle P, Stridsberg M \& Giwercman A 2003 Serum levels of 2,2',4,4',5, $5^{\prime}$-hexachlorobiphenyl (CB-153) in relation to markers of reproductive function in young males from the general Swedish population. Environmental Health Perspectives 111 409-413. (doi:10.1289/ehp.5767)

Rignell-Hydbom A, Lindh CH, Dillner J, Jonsson BA \& Rylander L 2012 A nested case-control study of intrauterine exposure to persistent organochlorine pollutants and the risk of hypospadias. PLOS ONE 7 e44767. (doi:10.1371/journal.pone.0044767)

Rozati R, Reddy PP, Reddanna P \& Mujtaba R 2002 Role of environmental estrogens in the deterioration of male factor fertility. Fertility and Sterility 78 1187-1194. (doi:10.1016/S0015-0282(02)04389-3)

Rylander L, Nilsson-Ehle P \& Hagmar L 2006 A simplified precise method for adjusting serum levels of persistent organohalogen pollutants to total serum lipids. Chemosphere 62 333-336. (doi:10.1016/j.chemosphere. 2005.04.107)

Safe S 1990 Polychlorinated biphenyls (PCBs), dibenzo-p-dioxins (PCDDs), dibenzofurans $(\mathrm{PCDFs})$, and related compounds: environmental and mechanistic considerations which support the development of toxic equivalency factors (TEFs). Critical Reviews in Toxicology 21 51-88. (doi:10.3109/10408449009089873)

Saxena MC, Siddiqui MK, Bhargava AK, Murti CR \& Kutty D 1981 Placental transfer of pesticides in humans. Archives of Toxicology 48 127-134. (doi:10.1007/BF00310482)
Seegal RF, Fitzgerald EF, Hills EA, Wolff MS, Haase RF, Todd AC, Parsons P, Molho ES, Higgins DS, Factor SA et al. 2011 Estimating the half-lives of PCB congeners in former capacitor workers measured over a 28-year interval. Journal of Exposure Science and Environmental Epidemiology 21 234-246. (doi:10.1038/jes.2010.3)

Sermondade N, Faure C, Fezeu L, Shayeb AG, Bonde JP, Jensen TK, Van Wely M, Cao J, Martini AC, Eskandar M et al. 2013 BMI in relation to sperm count: an updated systematic review and collaborative metaanalysis. Human Reproduction Update 19 221-231. (doi:10.1093/ humupd/dms050)

Sharpe RM, McKinnell C, Kivlin C \& Fisher JS 2003 Proliferation and functional maturation of Sertoli cells, and their relevance to disorders of testis function in adulthood. Reproduction 125 769-784. (doi:10.1530/ rep.0.1250769)

Skakkebaek NE, Rajpert-De Meyts E \& Main KM 2001 Testicular dysgenesis syndrome: an increasingly common developmental disorder with environmental aspects. Human Reproduction 16 972-978. (doi:10. 1093/humrep/16.5.972)

Sterne JA, White IR, Carlin JB, Spratt M, Royston P, Kenward MG, Wood AM \& Carpenter JR 2009 Multiple imputation for missing data in epidemiological and clinical research: potential and pitfalls. BMJ 338 b2393. (doi:10.1136/bmj.b2393)

Toft G, Rignell-Hydbom A, Tyrkiel E, Shvets M, Giwercman A, Lindh $\mathbf{C H}$, Pedersen HS, Ludwicki JK, Lesovoy V, Hagmar L et al. 2006 Semen quality and exposure to persistent organochlorine pollutants. Epidemiology 17 450-458. (doi:10.1097/01.ede.0000221769.41028.d2)

Toppari J, Larsen JC, Christiansen P, Giwercman A, Grandjean P, Guillette LJ Jr, Jegou B, Jensen TK, Jouannet P, Keiding N et al. 1996 Male reproductive health and environmental xenoestrogens. Environmental Health Perspectives 104 (Suppl 4) 741-803. (doi:10.1289/ehp. 96104s4741)

Toppari J \& Skakkebaek NE 1998 Sexual differentiation and environmental endocrine disrupters. Bailliere's Clinical Endocrinology and Metabolism 12 143-156. (doi:10.1016/S0950-351X(98)80529-6)

Vermeulen A, Verdonck L \& Kaufman JM 1999 A critical evaluation of simple methods for the estimation of free testosterone in serum. Journal of Clinical Endocrinology and Metabolism 84 3666-3667. (doi:10.1210/ jcem.84.10.6079)

Vested A, Ramlau-Hansen CH, Bonde JP, Thulstrup AM, Kristensen SL \& Toft G 2011 A comparison of conventional and computer-assisted semen analysis (CRISMAS software) using samples from 166 young Danish men. Asian Journal of Andrology 13 453-458. (doi:10.1038/aja.2011.14)

Vested A, Ramlau-Hansen CH, Olsen SF, Bonde JP, Kristensen SL, Halldorsson TI, Becher G, Haug LS, Ernst EH \& Toft G 2013 Associations of in utero exposure to perfluorinated alkyl acids with human semen quality and reproductive hormones in adult men. Environmental Health Perspectives 121 453-458. (doi:10.1289/ehp.1205118)

Virtanen HE, Koskenniemi JJ, Sundqvist E, Main KM, Kiviranta $H$, Tuomisto JT, Tuomisto J, Viluksela M, Vartiainen T, Skakkebaek NE et al. 2012 Associations between congenital cryptorchidism in newborn boys and levels of dioxins and PCBs in placenta. International Journal of Andrology 35 283-293. (doi:10.1111/j.1365-2605.2011.01233.x)

Wolff MS, Zeleniuch-Jacquotte A, Dubin N \& Toniolo P 2000 Risk of breast cancer and organochlorine exposure. Cancer Epidemiology, Biomarkers \& Prevention 9 271-277.

World Health Organization 1999 WHO Laboratory Manual for the Examination of Human Semen and Sperm-Cervical Mucus interaction, Geneva: World Health Organization, 4th ed. Cambridge, UK: Cambridge University Press.

Received 30 September 2013

First decision 22 October 2013

Revised manuscript received 25 August 2014

Accepted 4 September 2014 